\title{
Generating 24-Hour ECG, BP and Respiratory Signals with Realistic Linear and Nonlinear Clinical Characteristics Using a Nonlinear Model
}

\author{
GD Clifford $^{1}$, PE McSharry ${ }^{2,3,4}$ \\ ${ }^{1}$ Harvard-MIT Division of Health Sciences \& Technology, Cambridge MA, USA \\ ${ }^{2}$ Department of Engineering Science, University of Oxford, Oxford, UK \\ ${ }^{3}$ Mathematical Institute, University of Oxford, Oxford, UK \\ ${ }^{4}$ Centre for the Analysis of Time Series, London School of Economics, London, UK
}

\begin{abstract}
A nonlinear model for generating lifelike human ECG, blood pressure and respiratory signals is described. Each cycle of the model corresponds to one heart beat and the signals therefore exhibit beat-to-beat fluctuations by driving the model with a sequence of RR intervals. By using a modified version of entry no.201 of the CinC 2002 24-hour $R R$ interval generator challenge, (such that the user can specify the probability of ectopy or artefact) and coupling it to three ordinary differential equations, the model generates a 24-hour ECG signal. Using both standard linear metrics, and nonlinear long range statistics, the signal is shown to exhibit many of the known characteristics such as Respiratory Sinus Arrhythmia, Mayer waves and an overall diurnal rhythm.
\end{abstract}

The RR interval time series is modelled as a set of stationary states (joined by a transient heart rate overshoot) of differing lengths, mean heart rates (HR), LF/HF ratios and standard deviations. The length of time in each state is governed by a power law distribution with marked differences between waking and sleep states. The statistics of each RR time series segment (a state) can be fully specified by its mean (HR) and spectral distribution (LF/HF ratio).

The resultant ECG is shown to exhibit realistic QRSand QT-dispersions, $R$-S amplitude modulation and Respiratory Sinus Arrhythmia in the short term and normal values for nonlinear statistics (such as entropy) in the long term. By altering the parameters of the ECG model, introducing a heart-rate dependent delay (to simulate pulse transit time), and coupling the baseline to the long-term fluctuations of the 24 hour RR interval generator, realistic short and long range blood pressure fluctuations are shown to result. Together with seeded RR interval dynamics, the morphology of the signals can be fully specified by three parameters per feature and therefore a large range of different (deterministic) signals can be generated with fully known characteristics, to facilitate the testing of signal processing algorithms. Open source C, Matlab and Java programs for generating the model are available from Physionet.

\section{Introduction}

In order to effectively test the performance of signal processing algorithms for analysing biomedical signals, a noise-free signal is often desired [1]. A realistic artificial biomedical signal generator that is able to encompass the range of signals observed for both normal and abnormal subjects is therefore a useful tool. Furthermore, the ability to rapidly create a regenerateable time series enables a researcher to quickly prototype applications and test theories such as signal mixing and as a function of the model parameters such as sampling rate $[1,2]$.

Modifications of our published models for generating artificial 24-hour RR intervals [3] and ECG, blood pressure (BP) and respiration waveforms $[1,2]$ for generating 24-hour versions of these waveforms are presented. They are shown to have realistic properties; the signal possesses oscillations in morphological features and clinical parameters (such as QRS width, RS amplitude, RR and QTintervals) as well as statistical similarities with real data on many scales. Furthermore, the signals possess morphological appearances similar to that of a real waveforms and have a realistic inter-signal relationship.

\section{Methods}

\subsection{RR interval sequence}

During a 24 hour period, the HR tends to jump between different quantised states, relating to different physical and mental activity $[4,5]$, with different means $\bar{x}$, and variances, $\sigma^{2}$. The 24 hour tachogram is therefore built from a 
series of stationary states with prescribed means, variances and LF/HF ratios. See [2] for further explanation. Briefly, the long term oscillations (described in [3]) use empirical power-law distributions found in real data by BernaolaGalván et al. [5]. The length of time $\tau$ spent in a given state is governed by a power law distribution with $\tau=(u / \gamma)^{-\rho}$ where $\gamma=5466.8, \rho=2.2$ and $u$ is the uniform distribution $\sim U(\bar{x}, \sigma)$ with $\bar{x}=0, \sigma=1$. Fluctuations due to both a circadian rhythm (including wake-sleep and sleepwake transitions) and inter-sleep cycles are generated separately.

Circadian activity (high HR during wakefulness and low HR during sleep) leads to an approximately sinusoidal variation in baseline activity throughout the day [4]. During the wake stage, the mean RR interval is $\hat{\mu}_{R R} \sim U(0.7,1)$, with amplitude $U(0.075,2.075) \times$ $\left(\sin \left[\pi+\left(2 \pi / T_{c}\right) t\right]+\frac{2 r}{5}\right)$ and circadian period $T_{c} \sim$ $N(24,1) / 60^{2} \mathrm{~s}$ where $r$ is a zero mean, unit variance normal distribution $(r \sim N(0,1))$. During the sleep stage, the mean RR interval for a state is $U(0.7,1)+$ $\frac{1}{2} U(0.1,0.2)\left[1+\sin \left(2 \pi t / T_{s}\right)\right]$ and the period of the sleep cycle is $T_{s}=6 \times 10^{3} \mathrm{~s}$. The starting sleep time $T_{s}$ and sleep duration interval $T_{d}$ are given by $T_{s} \sim U(14,16) / 60^{2} \mathrm{~s}$ and $T_{d} \sim U(6,8) / 60^{2}$ s.

The size of transitions between different mean HRs, $\Delta \mu_{R R}$, are replicated using a distribution with a similar shape to that found from empirical observations $[3,5]$, $\Delta \mu_{R R}=\frac{1}{2} U(0.03,0.13)\left(1+e^{r} / 10\right) u /|u|$ with $r$ and $u$ are normal and uniform distributions where $r \sim N(0,1)$ and $u \sim U(0,1)$. The length of each transition between states is modelled by $\tau_{\text {trans }} \sim U(5,30)$. The over/undershoot and following compensatory return is replicated by superimposing a V-shaped bridge to span between the first and last RR interval of neighbouring states.

\subsection{The ECG \& BP and respiratory model}

The above RR interval model is then used to drive the angular frequency $\psi$ in the waveform model (described in detail in $[1,2,6]$ ) which has been modified to accept a vector of RR intervals. Briefly, the ECG is generated by a a fourth order Runge-Kutta integration [7] of three ODE's (Ordinary Differential Equations) in a 3D space $(x, y, z)$. Distinct points on the ECG, such as the P,Q,R,S and T are described by events corresponding to negative and positive Gaussian attractors/repellors in the $z$-direction. These events are placed at fixed angles along the unit circle given by $\theta_{P}, \theta_{Q}, \theta_{R}, \theta_{S}$ and $\theta_{T}$ which cause the trajectory to deviate from the $(x, y)$-plane in a Gaussian manner with amplitudes $a_{i}$, and widths $b_{i}$. The ODE's describing the dynamics are

$$
\begin{aligned}
& \dot{x}=\psi x-\omega y, \\
& \dot{y}=\psi y+\omega x,
\end{aligned}
$$

Table 1. ECG \& BP model parameters in (1). $\beta=\frac{\sqrt{\mathrm{HR}}}{60}$

\begin{tabular}{llllll}
\hline Index (i) & $P^{E C G}$ & $Q^{E C G}$ & $R^{E C G}$ & $S^{E C G}$ & $T^{E C G}$ \\
\hline Time (s) & -0.2 & -0.05 & 0 & 0.05 & 0.3 \\
$\theta_{i}$ (rads) & $\frac{-\pi}{3} \beta^{\frac{1}{2}}$ & $\frac{-\pi}{12} \beta$ & 0 & $\frac{\pi}{12} \beta$ & $\frac{\pi}{2} \beta^{\frac{1}{2}}$ \\
$a_{i}$ & 1.2 & -5.0 & 30.0 & -7.5 & 0.75 \\
$b_{i}$ & $0.25 \beta$ & $0.1 \beta$ & $0.1 \beta$ & $0.1 \beta$ & $0.4 \beta$ \\
\hline \hline Index (i) & $P^{B P}$ & $Q^{B P}$ & $R^{B P}$ & $S^{B P}$ & $T^{B P}$ \\
\hline Time (s) & 0.21 & 0.01 & 0 & 0.03 & 0.22 \\
$\theta_{i}$ (rads) & $\frac{-5 \pi}{12} \beta^{\frac{1}{2}}$ & $\frac{-\pi}{36} \beta$ & 0 & $\frac{\pi}{18} \beta$ & $\frac{4 \pi}{9} \beta^{\frac{1}{2}}$ \\
$a_{i}$ & 0 & 0 & 0.45 & 0.25 & 0.45 \\
$b_{i}$ & $0.25 \beta$ & $0.1 \beta$ & $0.3 \beta$ & $0.5 \beta$ & $0.3 \beta$ \\
\hline
\end{tabular}

$$
\dot{z}=-\sum_{i \in\{P, Q, R, S, T\}} a_{i} \Delta \theta_{i} e^{\left(-\Delta \theta_{i}^{2} / 2 b_{i}^{2}\right)}-\left(z-z_{0}\right),
$$

where $\psi=1-\sqrt{x^{2}+y^{2}}, \Delta \theta_{i}=\left(\theta-\theta_{i}\right) \bmod 2 \pi, \theta=$ atan2 $(y, x)$ and $\omega$ is the angular velocity of the trajectory as it moves around the limit cycle. The ECG is the motion of the trajectory in the $z$-direction. The BP waveform is generated in the same manner, but with different values of $\theta_{i}, a_{i}$ and $b_{i}$ (see table 1). The indexes for the BP no longer carry the same interpretation as with the ECG, with $i=R$ corresponding to the first peak and $i=S$ corresponding to the dichrotic notch. Figure 1 illustrates examples of these waveforms and their relationship.

Pulse transmission time (PTT) is therefore $2 \pi /\left(\theta_{Q}^{B P}-\right.$ $\left.\theta_{R}^{E C G}\right)$ seconds. PTT is inversely related to the pulse wave velocity down the artery which is known to be influenced by BP, HR, arterial compliance and hence age [8]. The effect of arterial compliance on PTT is not modelled. However, changes in PTT and BP as a function of HR (and therefore RR) interval are incorporated by adding an offset $\delta \theta$ to the $\theta_{i}$ at each beat such that $\delta \theta=\left(1-\left(\frac{60}{H R}\right)^{\frac{1}{2}} / 2\right) / 2 \pi$ to mimic the empirical results of Drinan et al. [8]. It is generally accepted that as BP falls, tension in the arterial wall falls and the PTT increases; and vice versa [9]. The systolic (peak) BP has therefore been linearly coupled to the mean HR (and hence inversely to the PTT).

The beat-to-beat variation in the waveform morphologies are induced by the variation in the integration step $d t$ to reflect changes in the RR interval; the time to complete one revolution around the attracting limit cycle in the $(x, y)$-plane. Shorter RR intervals (higher HR's) compress the waveform, resulting in shorter QT intervals and lower RS amplitudes (i.e. RSA). To mimic Bazett's law, a further compression factor is added; the $\theta_{i}$ are therefore premultiplied by a factor proportional to $\beta=\sqrt{\mathrm{HR}} / 60$.

The respiratory signal is simply the HF (parasympathetic) oscillation and is generated in the same manner as the RR intervals (using the inverse FT) using only the HF 


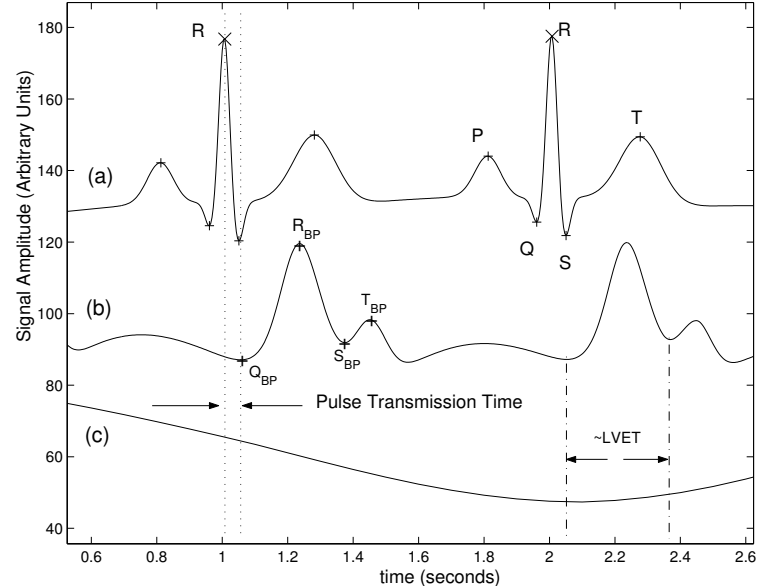

Figure 1. ECG (a), BP (b), and respiratory (c) signals labeled with PTT, LVET and P, Q, R, S, and T points, generated by integrating Eq. (1) over $\theta_{i}, a_{i}$ and $b_{i}$.

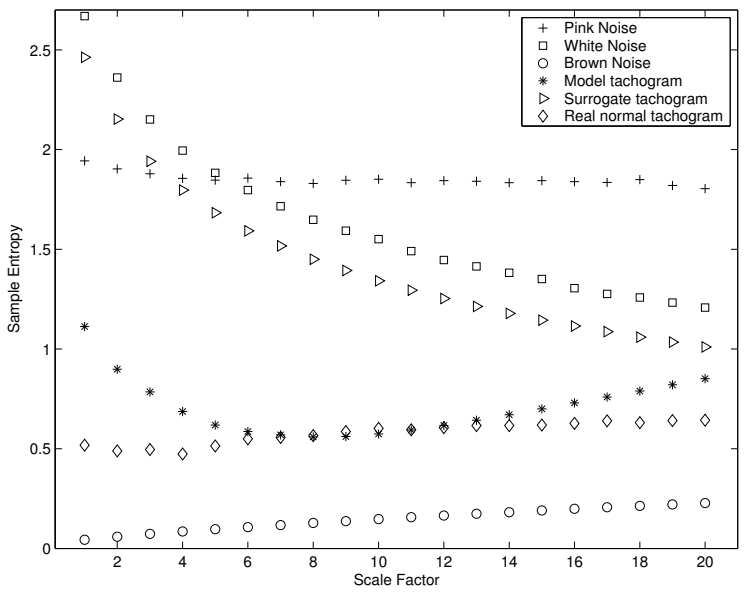

Figure 2. MSE results for model, surrogate, normal, pink noise, white noise and brown noise. See text for details

contribution.If, rather than being internally generated, the $\mathrm{RR}$ interval sequence is supplied as a vector, then the HF component of the signal is extracted by band pass filtering the signal between 0.1 and $0.5 \mathrm{~Hz}$. The sequence is then resampled to the desired output frequency.

\section{Results}

Linear metric results such as Realistic values for beatto-beat and HR-related amplitude modulation, QRS-width and QT-dispersion, and PR interval changes have already been reported for this model [2]. Since the BP signal is generated in the same manner as the ECG, the dynamics that affect the ECG also affect the BP signal. These have also been noted in a previous publication [1]. In particular we ensure that the random addition to the PTT results in a correlation $\rho$ between the PTT and RR interval that is no less than 0.7 as per Drinnan et al. [8]. The left ventricular ejection time (LVET) is proportional to the time between the onset of the BP waveform and the start of the reflected wave (the points $\theta_{Q}^{B P}$ and $\theta_{S}^{B P}$ in figure 1). The LVET has been shown to be longer for lower HR [10], a relationship that naturally emerges from the model presented here.

Although many nonlinear statistics have been applied to the ECG and BP waveforms, no clear consensus on the applicability of these metrics to the description of these waveforms has emerged [11]. We therefore confine our nonlinear analysis to the beat-to-beat interval dynamics. Detrended Fluctuation Analysis (DFA)[12] was employed in order to determine the long range correlations in the data. For one hundred random seeds, we found a pink noise-like scaling exponent of $\alpha=1.02 \pm 0.01$, which is in agreement for the $\alpha$ 's reported for healthy subjects [12]. Position randomised surrogates of these tachograms gave $\alpha=0.50 \pm 0.01$, a white noise scaling behaviour. The cross-over phenomenon at $\log _{10}(n) \approx 1.5$ is also observed.

A further complimentary scaling behaviour metric known as Multi Scale Entropy (MSE) [13] was also employed and compared to surrogates, real data, pink, white and brown noise (see figure 2). The scaling of the data was often patient specific (probably due to age differences [13]). For subject 040 of the NSRDB (illustrated in figure 2) the scaling behaviour is between pink and brown noise. This is consistent with the DFA scaling found for the same subject. The model scaling is consistent with the real data, particularly at the central scales. Similar results were found for BP intervals, with PTT having little effect on the scaling.

\section{Discussion}

Slight departures from normal behaviour of the MSE scaling for the model at extreme scales indicates that improvements could be made in the very short-term and very long-term behaviour of the model. In particular, the movement of the scaling towards pink and white noise behaviours for high (short) scales indicates that there is need for a more it Brownian-like complexity with a greater correlation between beat-to-beat intervals. This indicates that although the Fourier-inversion process to create the short sections of RR intervals produces a series of spectra with realistic $\mathrm{LF} / \mathrm{HF}$ values, the time-correlations of the data are not realistic enough. Although one may replace the short term RR interval creation process with a more realistic, physiology-based model (such as IPFM [14] or CVSIM [15]), it is not clear how the 24-hour distributions of RR interval model can be preserved.

A possible explanation for the MSE disparity at longer 
scales is the lack of intrinsic correlation between mean HR and $\mathrm{LF} / \mathrm{HF}$ ratios from segment to segment. In particular, during sleep, a subject will oscillate between deep and lighter sleep with slowly shortening and lengthening periods, which are often well correlated with shifts in the $\mathrm{LF} / \mathrm{HF}$ ratio and mean heart rates. This structure is not entirely mimicked in this model and only an overall shift in all the system parameters is seen. Furthermore, no explicit arousal mechanism exists in the model.

Although the ECG and BP signals exhibit a natural morphology (and changes) which can be adapted to any given patient, the respiratory signal lacks realism since it is too periodic. To introduce a more realistic morphology into the latter signal and mimic the asymmetry between exhalation and inhalation, it may prove useful to skew the signal in a manner proportional to the positive gradient. It should also be noted that no explicit delays have been built into the system to account for the pre-ejection period (PEP) and the ejection time (ET). However, a user-definable PTT relationship has been incorporated, and therefore the PEP and ET can be factored into this relationship if required.

\section{Conclusions}

The model presented in this paper comprises of two separate models; one for the beat-to-beat timing interval process and one for generating waveforms (such as the ECG, BP or respiratory signal) from these RR intervals. Model parameters are initialised using realistic distributions that can be modified so that different seeds produce different but repeatable biomedical signals. While the overall model is relatively complex, it summarises many of the important physiological control mechanisms that influence HR, BP and respiration over 24 hours and their short term intersignal relationships. Since each of the parameters of each model has a very clear physiological meaning, the performance of signal processing algorithms (such as how they respond to specific changes or contaminants) can be tested on a variety of artificial biomedical time series and may aid the teaching of the underlying mechanisms. Open source $\mathrm{C}$, Matlab and Java code for these models is available from Physionet [6].

\section{Acknowledgments}

This publication was made possible in part by Grant Number R01 EB001659 from the National Institute of Biomedical Imaging and Bioengineering (NIBIB) and by the Royal Academy of Engineers (RAE).

\section{References}

[1] Clifford GD, McSharry PE. A realistic coupled nonlinear artificial ECG, BP, and respiratory signal generator for assessing noise performance of biomedical signal processing algorithms. Proc of SPIE International Symposium on Fluctuations and Noise 2004;5467(34):290-301.

[2] McSharry PE, Clifford GD, Tarassenko L. A dynamical model for generating synthetic electrocardiogram signals. IEEE Trans Biomed Eng 2003;50(3):289-294.

[3] McSharry PE, Clifford GD, Tarassenko L. Method for generating an artificial RR tachogram of a typical healthy human over 24-hours. Computers in Cardiology 2002;29:225228.

[4] Brooks MC, Brooks NG. Rhythmic fluctuation of systemic arterial pressure and the cardiac myogenic contribution. Japan Sci. Soc. Press, Tokyo/Springer-Verlag, 1984. From Mechanisms of Blood Pressure Waves, (Ed. K. Miyakawa et al.) pp. 205-212.

[5] Bernaola-Galván P, Ivanov PC, Amaral LAN, Stanley HE. Scale invariance in the nonstationarity of the human heart rate. Phys Rev Lett 2001;87:168105.

[6] http://www.physionet.org/physiotools/ecgsyn/.

[7] Press W, Teukolsky S, Vetterlin W, Flannery B. Numerical Recipes in C: The art of scientific computing. Camb. Univ. Press, 1992. $2^{\text {nd }}$ Ed.

[8] Drinnan M.J. Allen J, Murray A. Relation between heart rate and pulse transit time during paced respiration. Physiol Meas 2001;22:425-432.

[9] Stradling JR, Barbour C, Glennon J, Langford BA, Crosby $\mathrm{JH}$. Which aspects of breathing during sleep influence the overnight fall of blood pressure in a community population? Thorax May 2000;55(5):393-398.

[10] Boudalis H. Systolic time intervals. Saunders, 1991. From Cardiac Output Measures, (Robertson JIS \& Birkenhager WH (Eds.).

[11] Kaplan DT, Furman MI, Pincus SM. Techniques for analyzing complexity in heart rate and beat-to-beat blood pressure signals. In Computers in Cardiology. IEEE Computer Society Press, 1990; 243-246.

[12] Peng CK, Mietus JE, Havlin S, Hausdorff JM, Stanley $\mathrm{HE}$, Goldberger AL. Long-range anticorrelations and nongaussian behavior of the heartbeat. Physical Review Letters 1993;70:1343-1346.

[13] Costa M, Goldberger AL, Peng CK. Multiscale entropy analysis of physiologic time series. Physical Review Letters 2002;89:062102.

[14] Brennan M, Palaniswami M, Kamen P. Distortion properties of the interval spectrum of IPFM generated heartbeats for heart raye variability analysis. IEEE Trans Biomed Eng November 2001;BME-48(11):1251-1263.

[15] Mukkamala R, Cohen RJ. A forward model-based validation of cardiovascular system identification. Am J Physiol Heart Circ Physiol 2001;281(6):2714-2730.

Address for correspondence:

Gari D. Clifford

Laboratory for Computational Physiology

Harvard-MIT Division of Health Sciences \& Technology

Rm E25-505, 45 Carleton St.,

Cambridge MA 02142 USA

gari@mit.edu 